\title{
The subtraction of mutually displaced Gaussian Schell-Model beams
}

\author{
J. Carlos G. de Sande ${ }^{1}$, Massimo Santarsiero², Gemma Piquero ${ }^{3}$, and Franco Gori² \\ 1 Departamento de Teoría de la Señal y Comunicaciones, Universidad Politécnica de Madrid, \\ Campos Sur, 28031 Madrid, Spain \\ 2 Dipartimento di Ingegneria, Università Roma Tre, via V. Volterra, 62; 00146 Rome, Italy \\ 3 Departamento de Óptica, Universidad Complutense de Madrid, 28040 Madrid, Spain
}

September 2, 2015

\begin{abstract}
Using recently derived results about the difference of two cross-spectral densities, we consider a source whose correlation function is the difference of two mutually displaced Gaussian Schellmodel cross-spectral densities. We examine the main features of this new cross-spectral density in terms of coherence and intensity distribution, both across the source plane and after free propagation.
\end{abstract}

\section{Introduction}

In classical optics, coherence theory [1-3] is a subject that continuously receives innovative contributions from countless authors, both within the scalar [4-11] and vectorial realm [12-21], including nonstationary states [22-26].

A basic tool in this theory is given by the cross-spectral density (CSD) [1,2]. Given the variety of physical situations one can encounter, different forms of the CSD are to be envisaged. From the mathematical viewpoint though, the CSD has to be a non-negative definite kernel [27], and this implies that its analytical structure cannot be chosen at will. Generally speaking, determining whether a kernel is endowed with the non-negativeness property is a difficult task, so that the risk of unphysical assumptions is to be tamed. Sufficient criteria to ensure correctness of alleged CSDs are quite useful. One such sufficient criterion was given not long ago [28,29], and proved its usefulness in several instances [30-32]. In the course of time, efforts were produced to understand operations suitable to lead to new CSD forms. In some recent papers [33,34] the possibility of constructing a new, genuine CSD by subtraction of two correlation functions was demonstrated. The subtraction of two coaxial Gaussian Schell-model (GSM) CSDs was studied in the case in which the shape-invariance condition is met [35]. As well known $[2,36]$ 
this means that the effects of propagation are limited to a scale expansion of the CSD accompanied by the onset of a curvature phase term. Accordingly, studying the coherence properties across a certain plane, e.g. the waist plane, is sufficient to enlighten the beam features through the whole space.

Here, we want to study a more general case of GSM CSDs subtraction. First, the two CSDs have different expansion coefficients, second, they are mutually displaced at any typical transverse plane except for planes in the far zone where a mutual displacement is replaced by different average inclination of the beam axes. In Section 2 the problem to be solved will be stated and, in Section 3, conditions for obtaining genuine CSD's from subtraction of two mutually displaced GSM CSDs will be obtained and an experimental way to obtain the described source will be proposed. The CSD properties at the source plane will be described in Section 4 while the properties of the CSD after propagation will be studied in Section 5. Finally, concluding remarks will be presented.

\section{Statement of the problem}

Let us start with a few words about the basic quantities we are going to use. The CSD at two points $\boldsymbol{\rho}_{1}$ and $\boldsymbol{\rho}_{2}$ of a given plane $z=$ const will be denoted by $W_{z}\left(\boldsymbol{\rho}_{1}, \boldsymbol{\rho}_{2}\right)$, omitting the explicit dependence from the temporal frequency. When the two points coincide, the CSD reduces to the optical intensity, i.e., $I_{z}(\boldsymbol{\rho})=W_{z}(\boldsymbol{\rho}, \boldsymbol{\rho})$. Furthermore, the spectral degree of coherence, is defined as [1]

$$
\mu_{z}\left(\boldsymbol{\rho}_{1}, \boldsymbol{\rho}_{2}\right)=\frac{W_{z}\left(\boldsymbol{\rho}_{1}, \boldsymbol{\rho}_{2}\right)}{\sqrt{I_{z}\left(\boldsymbol{\rho}_{1}\right) I_{z}\left(\boldsymbol{\rho}_{2}\right)}}
$$

Symbols like $W_{0}, I_{0}$, and $\mu_{0}$ will denote (with a slight notation abuse) the quantities $W_{z}$, $I_{z}$, and $\mu_{z}$ when the choice $z=0$ is done.

We shall study partially coherent fields in whose correlation function two GSM CSDs are subtracted from one another and determine conditions under which such subtraction operation gives rise to a genuine new CSD. Let us then assume the CSD across the source plane to be

$$
W_{0}\left(\boldsymbol{\rho}_{1}, \boldsymbol{\rho}_{2}\right)=W_{01}\left(\boldsymbol{\rho}_{1}, \boldsymbol{\rho}_{2}\right)-W_{02}\left(\boldsymbol{\rho}_{1}, \boldsymbol{\rho}_{2}\right),
$$

where [36]

$$
W_{0 j}\left(\boldsymbol{\rho}_{1}, \boldsymbol{\rho}_{2}\right)=B_{0 j} \exp \left[-\frac{\left(\boldsymbol{\rho}_{1}-\boldsymbol{v}_{j}\right)^{2}+\left(\boldsymbol{\rho}_{2}-\boldsymbol{v}_{j}\right)^{2}}{4 \sigma_{0 j}^{2}}-\frac{\left(\boldsymbol{\rho}_{1}-\boldsymbol{\rho}_{2}\right)^{2}}{2 \delta_{0 j}^{2}}\right], \quad(j=1,2),
$$

with positive constants $B_{0 j}$. The positive quantities $\sigma_{0 j}^{2}$ and $\delta_{0 j}^{2}$ denote intensity and coherence variances respectively. It is seen that the two contributions to $W_{0}$ are centered at possibly different positions specified by the vectors $\boldsymbol{v}_{j},(j=1,2)$. Nonetheless, since 
we can always move the coordinate origin we shall let $\boldsymbol{v}_{1}=0$ and write

$$
\begin{aligned}
W_{0}\left(\boldsymbol{\rho}_{1}, \boldsymbol{\rho}_{2}\right)= & B_{01} \exp \left[-\frac{\rho_{1}^{2}+\rho_{2}^{2}}{4 \sigma_{01}^{2}}-\frac{\left(\boldsymbol{\rho}_{1}-\boldsymbol{\rho}_{2}\right)^{2}}{2 \delta_{01}^{2}}\right] \\
& -B_{02} \exp \left[-\frac{\left(\boldsymbol{\rho}_{1}-\boldsymbol{v}_{2}\right)^{2}+\left(\boldsymbol{\rho}_{2}-\boldsymbol{v}_{2}\right)^{2}}{4 \sigma_{02}^{2}}-\frac{\left(\boldsymbol{\rho}_{1}-\boldsymbol{\rho}_{2}\right)^{2}}{2 \delta_{02}^{2}}\right] .
\end{aligned}
$$

The overall associated intensity is

$$
I_{0}(\boldsymbol{\rho})=B_{01} \exp \left(-\frac{\rho^{2}}{2 \sigma_{01}^{2}}\right)-B_{02} \exp \left[-\frac{\left(\boldsymbol{\rho}-\boldsymbol{v}_{2}\right)^{2}}{2 \sigma_{02}^{2}}\right] .
$$

An alternative and useful way of writing Eq. (4) is

$$
\begin{aligned}
W_{0}\left(\boldsymbol{\rho}_{1}, \boldsymbol{\rho}_{2}\right)= & B_{01} \exp \left[-P_{01}\left(\boldsymbol{\rho}_{1}+\boldsymbol{\rho}_{2}\right)^{2}-M_{01}\left(\boldsymbol{\rho}_{1}-\boldsymbol{\rho}_{2}\right)^{2}\right] \\
& -B_{02} \exp \left[-P_{02}\left(\boldsymbol{\rho}_{1}+\boldsymbol{\rho}_{2}-2 \boldsymbol{v}_{2}\right)^{2}-M_{02}\left(\boldsymbol{\rho}_{1}-\boldsymbol{\rho}_{2}\right)^{2}\right]
\end{aligned}
$$

where

$$
P_{0 j}=\frac{1}{8 \sigma_{0 j}^{2}} ; \quad M_{0 j}=\frac{1}{8 \sigma_{0 j}^{2}}+\frac{1}{2 \delta_{0 j}^{2}}, \quad(j=1,2) .
$$

Generally speaking, Eq. (4) does not represent a genuine CSD. A naive idea would be that of requiring that the intensity predicted by Eq. (5) be nowhere negative. Unfortunately, this condition alone does not guarantee that the CSD described by Eq. (4) is a genuine CSD. As a matter of fact, even if the optical intensity is non-negative throughout the whole space cases exist in which the modulus of the spectral degree of coherence can exceed one for certain pairs of points [34]. In the next Section, conditions on the parameters ensuring the CSD to be genuine will be derived.

\section{Conditions for obtaining a genuine CSD}

In [28] it has been shown that the validity of the expression of a CSD is warranted whenever the latter can be expressed through a superposition integral of the form

$$
W_{0}\left(\boldsymbol{\rho}_{1}, \boldsymbol{\rho}_{2}\right)=\int p(\boldsymbol{v}) H_{0}^{*}\left(\boldsymbol{\rho}_{1}, \boldsymbol{v}\right) H_{0}\left(\boldsymbol{\rho}_{2}, \boldsymbol{v}\right) \mathrm{d}^{2} v
$$

where $p$ is a non-negative function that will be referred to as the weight function, and $H_{0}$ is a suitable kernel. It has been also shown [29] that any valid CSD can be expressed through a representation of the form (8). We shall then use this representation as a guide for our analysis.

We shall begin by choosing $p$ of the form

$$
p(\boldsymbol{v})=p_{1}(\boldsymbol{v})-p_{2}(\boldsymbol{v})
$$


where $p_{1}$ and $p_{2}$ are two Gaussian functions, centered at the points 0 and $\boldsymbol{v}_{2}$, respectively, i.e.,

$$
p(\boldsymbol{v})=A_{1} \exp \left(-\gamma_{1} v^{2}\right)-A_{2} \exp \left[-\gamma_{2}\left(\boldsymbol{v}-\boldsymbol{v}_{\mathbf{2}}\right)^{2}\right]
$$

with positive constants $A_{1}, A_{2}$, and $\gamma_{1}, \gamma_{2}$. The kernel $H_{0}$ that we will use is itself a Gaussian function

$$
H_{0}(\boldsymbol{\rho}, \boldsymbol{v})=\exp \left[-\alpha(\boldsymbol{\rho}-\boldsymbol{v})^{2}\right],
$$

where $\alpha$ is a positive constant. We arrange the reference frame in such a way that $\boldsymbol{v}_{2}$ lies along the $x$-axis. Denoting by $u$ and $w$ the components of a typical $\boldsymbol{v}$ vector, the function $p$ then becomes

$$
p(u, w)=A_{1} \exp \left\{-\gamma_{1}\left(u^{2}+w^{2}\right)\right\}-A_{2} \exp \left\{-\gamma_{2}\left[\left(u-u_{2}\right)^{2}+w^{2}\right]\right\} .
$$

We now derive the conditions to be satisfied by $A_{j}$ and $\gamma_{j},(j=1,2)$ in order to have $p(\boldsymbol{v}) \geq 0$ for any $\boldsymbol{v}$. First, in order to ensure a non-negative $p$ when $|\boldsymbol{v}|$ tends to infinity the following condition has to be satisfied

$$
\gamma_{2}>\gamma_{1}
$$

Let us now discuss the behavior of $p(\boldsymbol{v})$ for finite values of $\boldsymbol{v}$. In qualitative terms, the presence of the second Gaussian term digs a hole in the surface $p(u, w)$. An example is given in Fig. 1, where a density plot of Eq. (12) is shown for $A_{1}=1, \gamma_{1}=0.5, A_{2}=0.95$, $\gamma_{2}=0.75$ and $u_{2}=0.1$. All the parameters are in arbitrary units throughout the whole text and figures.

The bottom of the hole digged in the surface $p(u, w)$ is located at a minimum for $p(u, w)$. At that point, two conditions have to be met. First, both partial derivatives of $p(u, w)$ have to vanish. Second, the Hessian must be positive. We can dispose of the second condition, being evident by direct inspection that the point (at a finite distance from the origin) where both first partial derivatives vanish corresponds to a minimum. The vanishing of the partial derivatives gives the two equations

$$
\begin{gathered}
\frac{\partial p(u, w)}{\partial u}=-2 A_{1} \gamma_{1} u e^{-\gamma_{1}\left(u^{2}+w^{2}\right)}+2 A_{2} \gamma_{2}\left(u-u_{2}\right) e^{-\gamma_{2}\left[\left(u-u_{2}\right)^{2}+w^{2}\right]}=0, \\
\frac{\partial p(u, w)}{\partial w}=-2 A_{1} \gamma_{1} w e^{-\gamma_{1}\left[u^{2}+w^{2}\right]}+2 A_{2} \gamma_{2} w e^{-\gamma_{2}\left[\left(u-u_{2}\right)^{2}+w^{2}\right]}=0 .
\end{gathered}
$$

In addition, since $p(\boldsymbol{v})$ is to be non-negative everywhere, its minimum is required to be non-negative as well. Equation (15) is satisfied along the whole $u$-axis (line $w=0$ ). Letting $w=0$ we derive from Eq. (14)

$$
A_{1} \gamma_{1} u e^{-\gamma_{1} u^{2}}=A_{2} \gamma_{2}\left(u-u_{2}\right) e^{-\gamma_{2}\left[\left(u-u_{2}\right)^{2}\right]} .
$$

Let us refer to the limiting case in which the minimum of $p$ is a zero. Then, Eq. (12) along the line $w=0$ gives

$$
A_{1} \exp \left(-\gamma_{1} u^{2}\right)=A_{2} \exp \left[-\gamma_{2}\left(u-u_{2}\right)^{2}\right]
$$




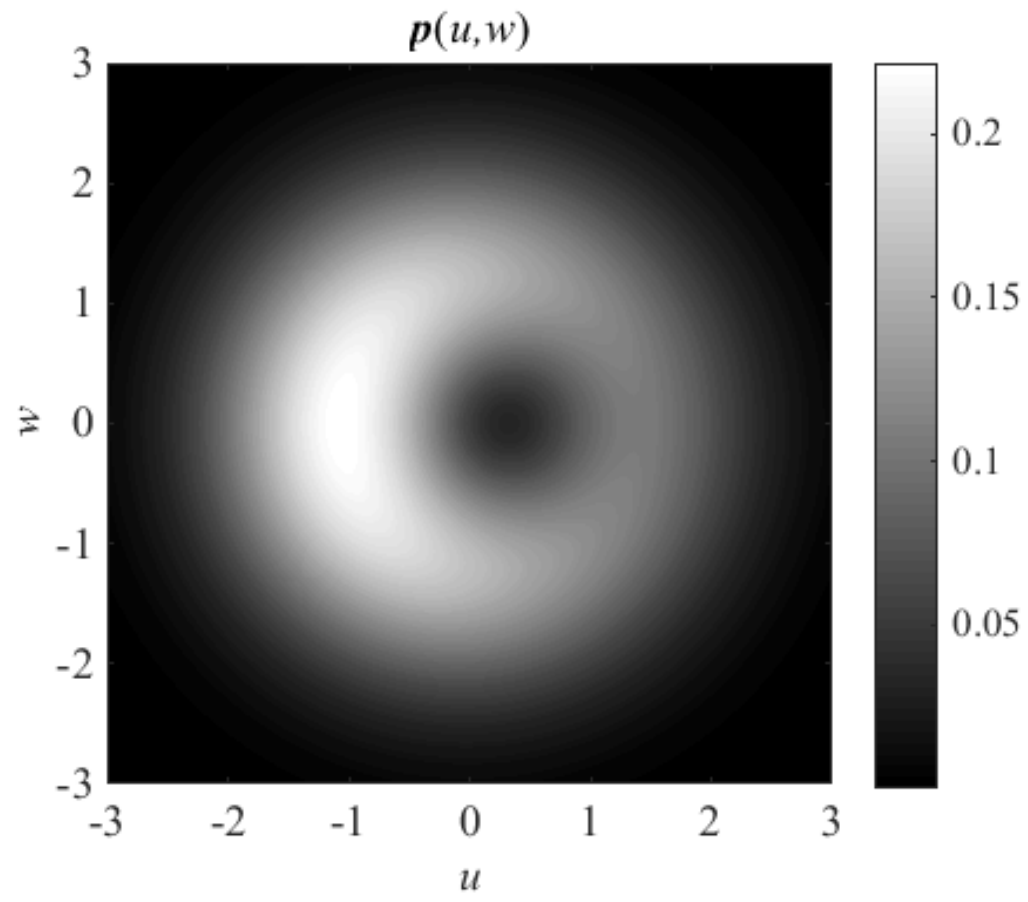

Figure 1: Weight function associated with Eq. (12), with $A_{1}=1, \gamma_{1}=0.5, A_{2}=0.95$, $\gamma_{2}=0.75$ and $u_{2}=0.1$.

which, when inserted into Eq. (14) (at $w=0$ ), leads to

$$
\gamma_{1} u=\gamma_{2}\left(u-u_{2}\right)
$$

or

$$
u=\frac{\gamma_{2} u_{2}}{\gamma_{2}-\gamma_{1}}
$$

When this value of $v$ is inserted into Eq. (17) we obtain

$$
A_{1}=A_{2} \exp \left(\frac{\gamma_{1} \gamma_{2}}{\gamma_{2}-\gamma_{1}} u_{2}^{2}\right)
$$

Obviously, if $A_{1}$ is greater than the right-hand side of this equation, the minimum of $p$ will be positive. In conclusion, the following conditions guarantee that Eqs. (8), (10), and (11) furnish a genuine CSD

$$
A_{1} \geq A_{2} \exp \left(\frac{\gamma_{1} \gamma_{2}}{\gamma_{2}-\gamma_{1}} u_{2}^{2}\right) ; \quad \gamma_{2}>\gamma_{1} \text {. }
$$

We note that, when $\boldsymbol{v}_{2}=0$, such conditions reduce to those already obtained in [34], where the case of the subtraction of two centered GSM CSDs was studied.

Behaviors of the weight function $p(u, w=0)$ can be observed in Fig. 2 for several values of the displacement $u_{2}$ and the maximum allowed value of $A_{2}(\mathrm{a})$, and for $u_{2}=0.1$ and several values of $A_{2}(\mathrm{~b})$. 
(a)

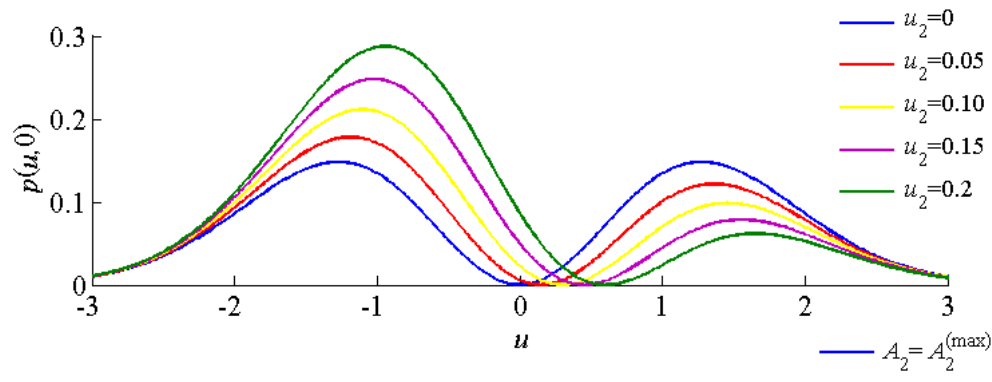

(b)

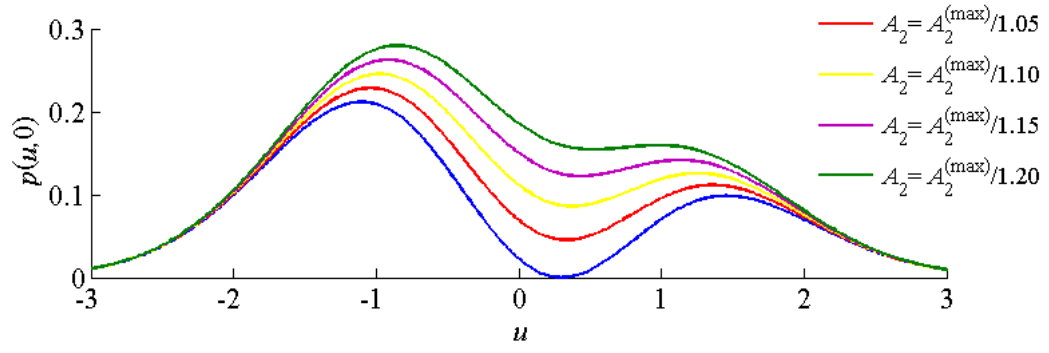

Figure 2: Weight function associated with Eq. (12), with $A_{1}=1, \gamma_{1}=0.5, \gamma_{2}=0.75$. (a) as a function of $u$ with $w=0, A_{2}=A_{2}^{(\max )}=A_{1} \exp \left(-\frac{\gamma_{1} \gamma_{2}}{\gamma_{2}-\gamma_{1}} u_{2}^{2}\right)$ and several values of $u_{2}$; (b) as a function of $u$ with $w=0, u_{2}=0.1$ and several values of $A_{2}$.

Before concluding this section, we want to suggest an experimental procedure aimed at producing partially coherent sources of the type discussed up to now. Production of several partially coherent fields with prescribed CSD have been obtained in recent times [10] using sophisticated methods of spatial light modulation. In our case, as we shall see, a simple optical system can be employed.

The basic idea comes directly from the integral representation given in Eq. (8). Imagine a partially coherent field described by a certain CSD $W_{\text {in }}\left(\boldsymbol{v}, \boldsymbol{v}^{\prime}\right)$ is sent to the input plane of a linear system characterized by a coherent impulse response $H_{0}(\boldsymbol{\rho}, \boldsymbol{v})$. The CSD across the output plane of the system will be [2]

$$
W_{0}\left(\boldsymbol{\rho}_{1}, \boldsymbol{\rho}_{2}\right)=\iint W_{\mathrm{in}}\left(\boldsymbol{v}, \boldsymbol{v}^{\prime}\right) H_{0}^{*}\left(\boldsymbol{\rho}_{1}, \boldsymbol{v}\right) H_{0}\left(\boldsymbol{\rho}_{2}, \boldsymbol{v}^{\prime}\right) \mathrm{d}^{2} v \mathrm{~d}^{2} v^{\prime},
$$

both integrals being extended to the source plane. Now, we introduce the hypothesis that the input field is spatially incoherent, so that its CSD is specified by the delta-like structure $W_{\text {in }}\left(\boldsymbol{v}, \boldsymbol{v}^{\prime}\right)=p(\boldsymbol{v}) \delta\left(\boldsymbol{v}-\boldsymbol{v}^{\prime}\right)$ where $p(\boldsymbol{v})$ is the intensity distribution. On inserting this condition into Eq. (22) we obtain the same relation as Eq. (8). Therefore, we can say that the CSD described by Eq. (8) is displayed at the output plane of an optical system fed by an intensity distribution of the form given in Eq. (10) and endowed with the coherent impulse response in Eq. (11).

The optical device can simply be the $4 f$ telescopic system of Fig. 3 [37]. The incoherent intensity distribution (10) (produced through uniform illumination of a suitable filter) is put across the input plane (left). In the pupil plane (common focal plane of the two 


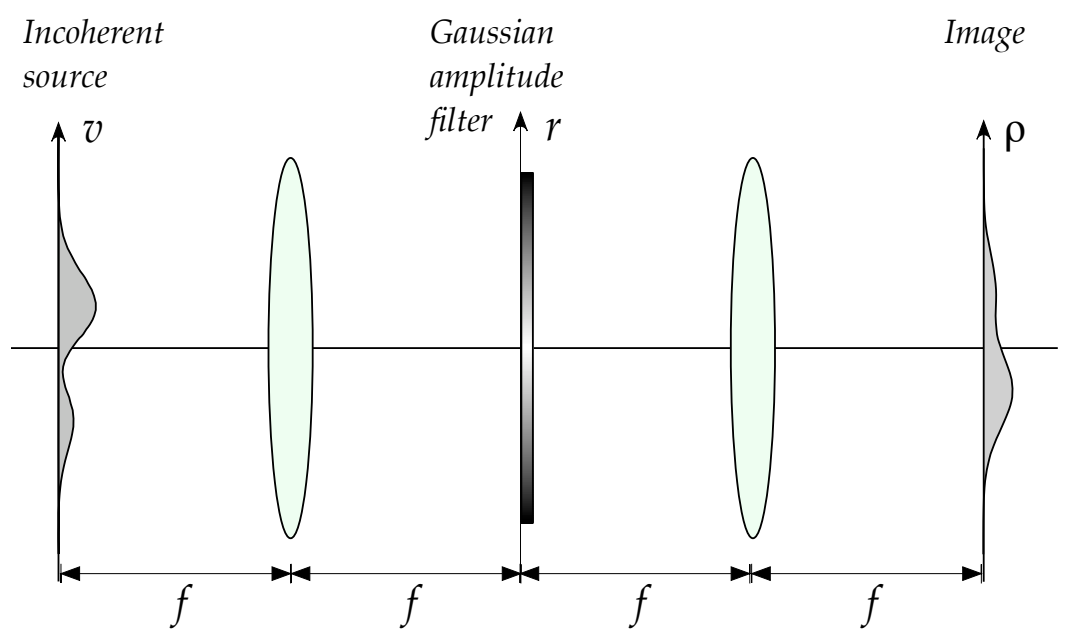

Figure 3: Schematic of a $4 f$ telescopic system.

lenses) a Gaussian filter with transmission function $\exp \left[-\pi^{2} r^{2} /\left(\lambda^{2} f^{2} \alpha\right)\right]$ is put to produce the impulse response (11). The synthesized source appears at the exit plane (right) as the image of the system.

\section{Properties of the CSD across the source plane}

According to Eqs. (8) and (10), the CSD at the source plane $(z=0)$ is given by

$$
\begin{aligned}
W_{0}\left(\boldsymbol{\rho}_{1}, \boldsymbol{\rho}_{2}\right)= & A_{1} \int \exp \left(-\gamma_{1} v^{2}\right) \exp \left[-\alpha\left(\boldsymbol{\rho}_{1}-\boldsymbol{v}\right)^{2}\right] \exp \left[-\alpha\left(\boldsymbol{\rho}_{2}-\boldsymbol{v}\right)^{2}\right] d^{2} v \\
& -A_{2} \int \exp \left[-\gamma_{2}\left(\boldsymbol{v}-\boldsymbol{v}_{2}\right)^{2}\right] \exp \left[-\alpha\left(\boldsymbol{\rho}_{1}-\boldsymbol{v}\right)^{2}\right] \exp \left[-\alpha\left(\boldsymbol{\rho}_{2}-\boldsymbol{v}\right)^{2}\right] d^{2} v
\end{aligned}
$$

where the constants satisfy conditions (21). By straightforward evaluation of the integrals we obtain

$$
\begin{aligned}
& W_{0}\left(\boldsymbol{\rho}_{1}, \boldsymbol{\rho}_{2}\right)=\frac{\pi A_{1}}{\gamma_{1}+2 \alpha} \exp \left[-\frac{\alpha \gamma_{1}+\alpha^{2}}{\gamma_{1}+2 \alpha}\left(\rho_{1}^{2}+\rho_{2}^{2}\right)+\frac{2 \alpha^{2}}{\gamma_{1}+2 \alpha} \boldsymbol{\rho}_{1} \cdot \boldsymbol{\rho}_{2}\right] \\
& -\frac{\pi A_{2}}{\gamma_{2}+2 \alpha} \exp \left[-\frac{\alpha \gamma_{2}+\alpha^{2}}{\gamma_{2}+2 \alpha}\left[\left(\boldsymbol{\rho}_{1}-\boldsymbol{v}_{2}\right)^{2}+\left(\boldsymbol{\rho}_{2}-\boldsymbol{v}_{2}\right)^{2}\right]+\frac{2 \alpha^{2}}{\gamma_{2}+2 \alpha}\left(\boldsymbol{\rho}_{1}-\boldsymbol{v}_{2}\right) \cdot\left(\boldsymbol{\rho}_{2}-\boldsymbol{v}_{2}\right)\right] .
\end{aligned}
$$

Therefore, each component of the overall CSD is of the GSM form. On using the following relations:

$$
B_{0 j}=A_{0 j} \frac{\pi}{\gamma_{j}+2 \alpha} ; \quad \frac{1}{4 \sigma_{0 j}^{2}}=\frac{\alpha \gamma_{j}}{\gamma_{j}+2 \alpha} ; \quad \frac{1}{2 \delta_{0 j}^{2}}=\frac{\alpha^{2}}{\gamma_{j}+2 \alpha}, \quad(j=1,2)
$$


Eq. (24) is easily transformed into Eq. (4).

Furthermore, using the the definitions in Eq. (7), it turns out that

$$
P_{0 j}=\frac{\alpha \gamma_{j}}{2 \gamma_{j}+4 \alpha}, \quad(j=1,2) ; \quad M_{01}=M_{02}=M=\alpha / 2 .
$$

Note that the choice of a kernel $H_{0}$ in the form given by Eq. (11) gives rise to the class of sources described in Theorem 2 of Ref. [34], (i.e., the difference of two GSM sources with the same values of the parameter $M$ ) with the only difference that now we are considering the possibility of a lateral displacement of the two sources. This implies that the inequalities in Eq. (21) represent necessary and sufficient conditions in order for Eq. (4) to express a genuine CSD.

Using the relations in Eq. (25) and in Eq. (26), inequalities (21) can be transformed into

$$
B_{01} \geq B_{02} \frac{M-P_{01}}{M-P_{02}} \exp \left(\frac{4 P_{01} P_{02}}{P_{02}-P_{01}} u_{2}^{2}\right) ; \quad P_{02}>P_{01} .
$$

or, in terms of intensity and coherence variances, as

$$
B_{01} \geq B_{02}\left(\frac{\delta_{02}}{\delta_{01}}\right)^{2} \exp \left[\frac{u_{2}^{2}}{2\left(\sigma_{01}^{2}-\sigma_{02}^{2}\right)}\right] ; \quad \delta_{02}>\delta_{01},
$$

where, taking Eq. (7) into account, $\sigma_{01}>\sigma_{02}$.

It is interesting to enquire about the spectral degree of coherence. In the case of shape-invariant subtraction of coaxial GSM CSDs [35] it was found that, for any choice of $\boldsymbol{\rho}_{1}$, there are points $\boldsymbol{\rho}_{2}$ such that the degree of coherence between $\boldsymbol{\rho}_{1}$ and $\boldsymbol{\rho}_{2}$ vanishes. In particular, there is a circle over which we can move $\boldsymbol{\rho}_{2}$ always obtaining a zero degree of coherence with respect to point $\boldsymbol{\rho}_{1}$. Such a circle is not centered at $\boldsymbol{\rho}_{1}$ except in the case $\boldsymbol{\rho}_{1}=0$. We wonder whether, for a typical value of $\boldsymbol{\rho}_{1}$, positions $\boldsymbol{\rho}_{2}$ can be found such that $\mu\left(\boldsymbol{\rho}_{1}, \boldsymbol{\rho}_{2}\right)=0$. In other words, using Eq. (6), we wonder whether we can solve the equation

$$
\begin{aligned}
& B_{01} \exp \left[-P_{01}\left(\boldsymbol{\rho}_{1}+\boldsymbol{\rho}_{2}\right)^{2}-M_{01}\left(\boldsymbol{\rho}_{1}-\boldsymbol{\rho}_{2}\right)^{2}\right] \\
& =B_{02} \exp \left[-P_{02}\left(\boldsymbol{\rho}_{1}+\boldsymbol{\rho}_{2}-2 \boldsymbol{v}_{2}\right)^{2}-M_{02}\left(\boldsymbol{\rho}_{1}-\boldsymbol{\rho}_{2}\right)^{2}\right],
\end{aligned}
$$

or, dividing side by side and passing to the logarithms,

$$
\ln \beta=P_{01}\left(\boldsymbol{\rho}_{1}+\boldsymbol{\rho}_{2}\right)^{2}-P_{02}\left(\boldsymbol{\rho}_{1}+\boldsymbol{\rho}_{2}-2 \boldsymbol{v}_{2}\right)^{2},
$$

where we let

$$
B_{01} / B_{02}=\beta,
$$

and the equality $M_{01}=M_{02}$ has been taken into account. We take, as before, $w_{2}=0$ and $u_{2} \neq 0$. By simple manipulation Eq. (30) can be changed into

$$
\left(\boldsymbol{\rho}_{1}+\boldsymbol{\rho}_{2}-\frac{2 P_{02}}{P_{02}-P_{01}} \boldsymbol{v}_{2}\right)^{2}=\frac{4 P_{01} P_{02}}{\left(P_{02}-P_{01}\right)^{2}} u_{2}^{2}-\frac{\ln \beta}{P_{02}-P_{01}} .
$$


This equation requires its left-hand side to be non-negative, and this happens whenever

$$
u_{2}^{2}>\frac{\left(P_{02}-P_{01}\right) \ln \beta}{4 P_{01} P_{02}} .
$$

The above inequality can be expressed as

$$
B_{01}<B_{02} \exp \left(\frac{4 P_{01} P_{02}}{P_{02}-P_{01}} u_{2}^{2}\right)
$$

which is clearly incompatible with the inequalities in Eq. (27). The conclusion is that no point pairs exist for which the degree of correlation is zero.

\section{Propagation}

Starting from the expression (6) for the CSD across the plane $z=0$ we can write the CSD at a plane $z=$ const $\neq 0$ upon paraxial propagation [36] as

$$
\begin{gathered}
W_{z}\left(\boldsymbol{\rho}_{1}, \boldsymbol{\rho}_{2}\right)=B_{z 1} \exp \left[\frac{i k}{2 R_{z 1}}\left(\rho_{2}^{2}-\rho_{1}^{2}\right)-P_{z 1}\left(\boldsymbol{\rho}_{1}+\boldsymbol{\rho}_{2}\right)^{2}-M_{z 1}\left(\boldsymbol{\rho}_{1}-\boldsymbol{\rho}_{2}\right)^{2}\right] \\
-B_{z 2} \exp \left\{\frac{i k}{2 R_{z 2}}\left[\rho_{2}^{2}-\rho_{1}^{2}-2\left(\boldsymbol{\rho}_{2}-\boldsymbol{\rho}_{1}\right) \cdot \boldsymbol{v}_{2}\right]\right. \\
\left.-P_{z 2}\left(\boldsymbol{\rho}_{1}+\boldsymbol{\rho}_{2}-2 \boldsymbol{v}_{2}\right)^{2}-M_{z 2}\left(\boldsymbol{\rho}_{1}-\boldsymbol{\rho}_{2}\right)^{2}\right\}
\end{gathered}
$$

where the parameters in the right-hand side vary according to the formulas

$$
B_{z j}=\frac{B_{0 j}}{F_{j}^{2}(z)} ; \quad P_{z j}=\frac{P_{0 j}}{F_{j}^{2}(z)} ; \quad M_{z j}=\frac{M_{0 j}}{F_{j}^{2}(z)} ; \quad R_{z j}=z\left[1+\left(\frac{\pi s_{0 j}^{2}}{\lambda z}\right)^{2}\right] ; \quad(j=1,2),
$$

$s_{0 j}$ being the spot-size of the underlying modes [38], whose square is given by

$$
s_{0 j}^{2}=\frac{1}{\sqrt{4 P_{0 j} M_{0 j}}}, \quad(j=1,2),
$$

and is related to the Rayleigh range of each of the two GSM beams, namely [39]

$$
z_{R j}=\frac{\pi s_{0 j}^{2}}{\lambda}, \quad(j=1,2) .
$$

The functions $F_{j}(z)$ are given by

$$
F_{j}(z)=\sqrt{1+\left(\frac{\lambda z}{\pi s_{0 j}^{2}}\right)^{2}}=\sqrt{1+\left(\frac{z}{z_{R j}}\right)^{2}}, \quad(j=1,2),
$$




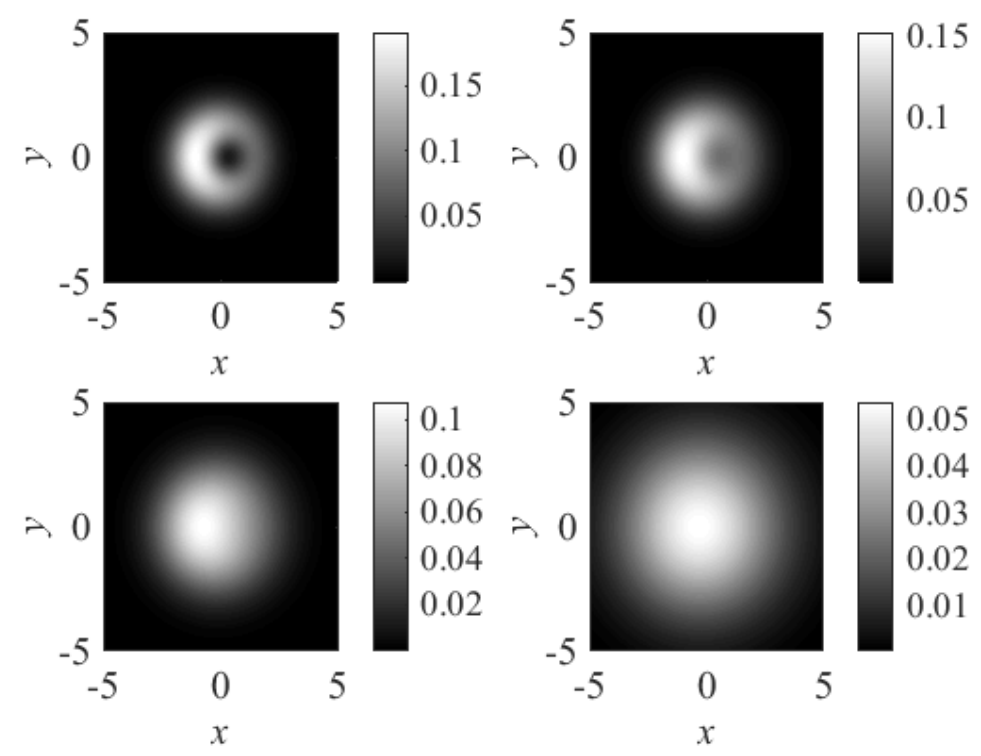

Figure 4: Intensity across transverse planes at $z=0, z=0.5 z_{R 1}, z=z_{R 1}$, and $z=2 z_{R 1}$ from left to right and from top to bottom. Furthermore, $\sigma_{01}=1, \sigma_{02}=0.85, \delta_{02}=0.5$, $u_{2}=0.1, B_{01}=1$, and $B_{02}=B_{02}^{(\max )}$.

and play the role of expansion factors. Taking into account the relations between the $P$, $M$ parameters and the $\sigma, \delta$ quantities, it is seen in fact that both the intensity and the coherence spot-sizes increase upon propagation, being multiplied by $F_{j}(z)$. The mutual displacement of the two component CSDs, represented by the vector $\boldsymbol{v}_{2}$ though remains unchanged, and becomes less and less significant when propagation proceeds. A few examples are given in Figs. 4, 5, and 6, where plots of the intensity profile and of the absolute value of the degree of coherence are shown. The source parameters are $\sigma_{01}=1$, $\sigma_{02}=0.85, \delta_{02}=0.5, u_{2}=0.1, B_{01}=1$, (note that $\delta_{01} \simeq 0.4941$ is determined by Eq. (26) and the definition of $M_{0 j}$ ), while $B_{02}$ takes the maximum value allowed by Eq. (28), i.e.,

$$
B_{02}^{(\max )}=\left(\frac{\delta_{01}}{\delta_{02}}\right)^{2} \exp \left[\frac{-u_{2}^{2}}{2\left(\sigma_{01}^{2}-\sigma_{02}^{2}\right)}\right] .
$$

It is worth considering explicitly the behavior in the far zone. There, the CSD is known to be proportional (up to scale and amplitude factors) to the Fourier transform of $W_{0}$ evaluated at $\left(\boldsymbol{\nu}_{1},-\boldsymbol{\nu}_{2}\right)$, where $\boldsymbol{\nu}_{1}$ and $\boldsymbol{\nu}_{2}$ are the conjugate variables of $\boldsymbol{\rho}_{1}$ and $\boldsymbol{\rho}_{2}$, respectively. Up to this convention we write

$$
W^{(\infty)}\left(\boldsymbol{\nu}_{1}, \boldsymbol{\nu}_{2}\right)=\widetilde{W}_{0}\left(\boldsymbol{\nu}_{1},-\boldsymbol{\nu}_{2}\right)=\iint W_{0}\left(\boldsymbol{\rho}_{1}, \boldsymbol{\rho}_{2}\right) e^{2 \pi i\left(\boldsymbol{\rho}_{1} \cdot \boldsymbol{\nu}_{1}-\boldsymbol{\rho}_{2} \cdot \boldsymbol{\nu}_{2}\right)} \mathrm{d}^{2} \rho_{1} \mathrm{~d}^{2} \rho_{2}
$$



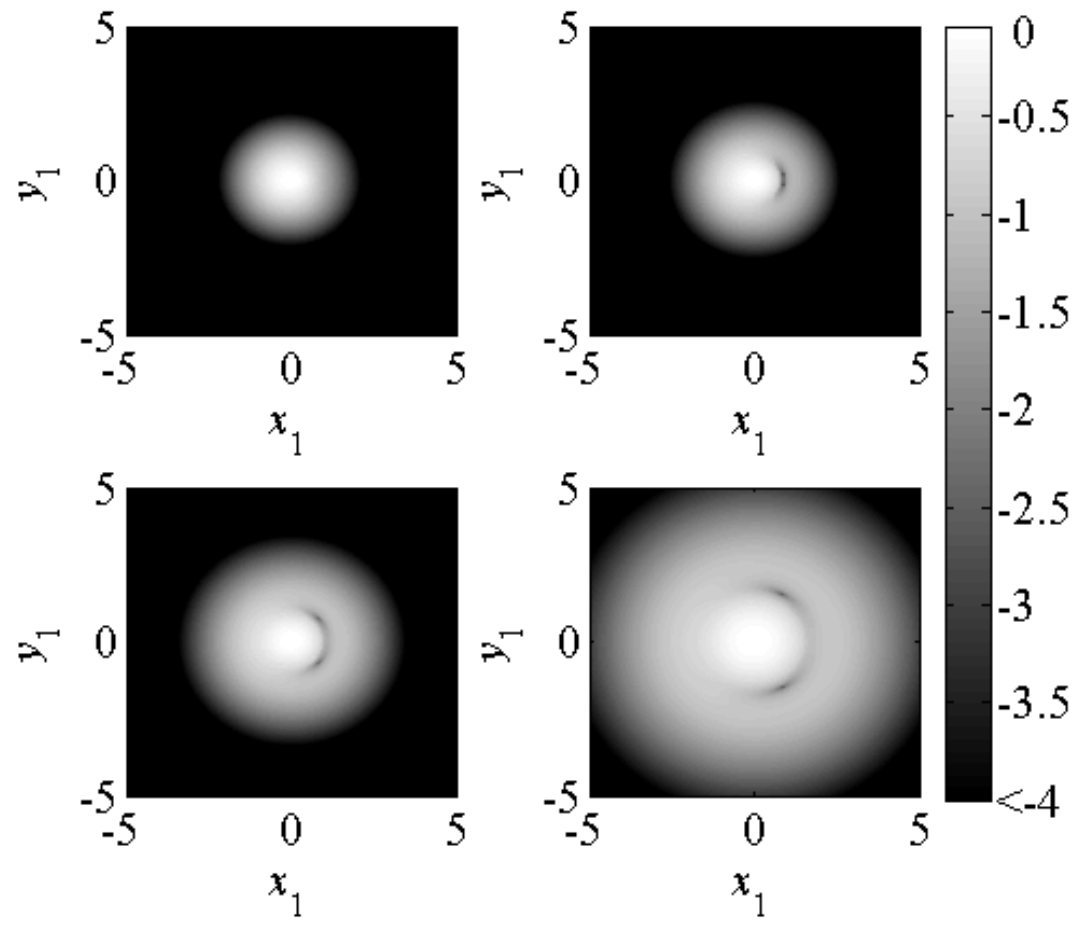

Figure 5: Absolute value of the degree of coherence $\mu\left(\boldsymbol{\rho}_{1}, \boldsymbol{\rho}_{2}=0\right)$, in logarithmic scale, across transverse planes at $z=0, z=0.5 z_{R 1}, z=z_{R 1}$, and $z=2 z_{R 1}$ from left to right and from top to bottom. Furthermore, $\sigma_{01}=1, \sigma_{02}=0.85, \delta_{02}=0.5, u_{2}=0.1, B_{01}=1$, and $B_{02}=B_{02}^{(\max )}$. 

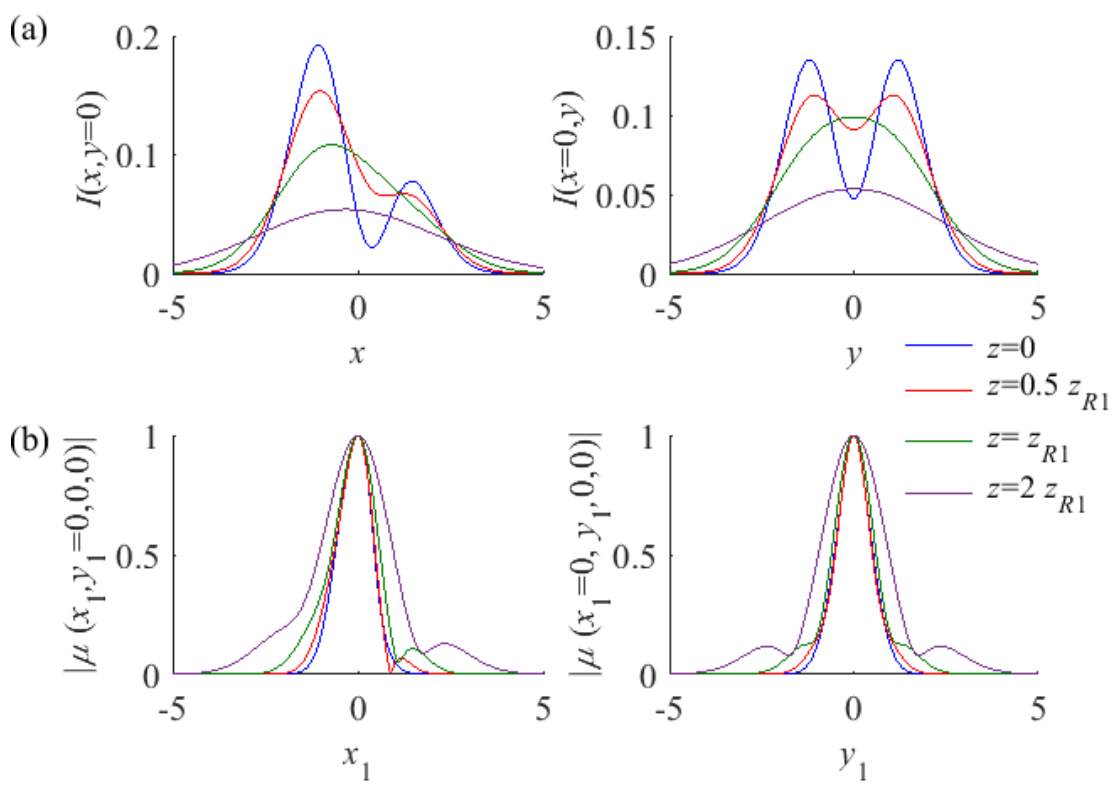

Figure 6: Intensity (a) and modulus of the degree of coherence (b) along the $x$ and $y$ direction at different propagation distances. Other parameters are $\sigma_{01}=1, \sigma_{02}=0.85$, $\delta_{02}=0.5, u_{2}=0.1, B_{01}=1$, and $B_{02}=B_{02}^{(\max )}$.

On replacing $W_{0}$ in the integral by the expression given by Eq. (6), it turns out that

$$
\begin{aligned}
& W^{(\infty)}\left(\boldsymbol{\nu}_{1}, \boldsymbol{\nu}_{2}\right)=\frac{\pi B_{01}}{M P_{01}} \exp \left[-\frac{\pi^{2}}{2 M}\left(\nu_{1}^{2}+\nu_{2}^{2}\right)\right] \exp \left[\frac{-\pi^{2}\left(M-P_{01}\right)}{4 M P_{01}}\left(\boldsymbol{\nu}_{1}-\boldsymbol{\nu}_{2}\right)^{2}\right] \\
& -\frac{\pi B_{02}}{M P_{02}} \exp \left[-\frac{\pi^{2}}{2 M}\left(\nu_{1}^{2}+\nu_{2}^{2}\right)\right] \exp \left[-\frac{\pi^{2}\left(M-P_{02}\right)}{4 M P_{02}}\left(\boldsymbol{\nu}_{1}-\boldsymbol{\nu}_{2}\right)^{2}\right] \exp \left[\mathrm{i}\left(\boldsymbol{\nu}_{1}-\boldsymbol{\nu}_{2}\right) \cdot \boldsymbol{v}_{2}\right],
\end{aligned}
$$

which can be written as

$$
W^{(\infty)}\left(\boldsymbol{\nu}_{1}, \boldsymbol{\nu}_{2}\right)=\sqrt{I^{(\infty)}\left(\boldsymbol{\nu}_{1}\right)} \sqrt{I^{(\infty)}\left(\boldsymbol{\nu}_{2}\right)} \mu^{(\infty)}\left(\boldsymbol{\nu}_{1}-\boldsymbol{\nu}_{2}\right)
$$

with

$$
I^{(\infty)}(\boldsymbol{\nu})=\frac{\pi}{M}\left(\frac{B_{01}}{P_{01}}-\frac{B_{02}}{P_{02}}\right) \exp \left(-\frac{\pi \nu^{2}}{M}\right)
$$

and

$$
\begin{aligned}
\mu^{(\infty)}(\boldsymbol{\nu})= & \frac{B_{01} P_{02} \exp \left(-\frac{\pi^{2} \nu^{2}}{4 P_{01}}\right)-B_{02} P_{01} \exp \left(-\frac{\pi^{2} \nu^{2}}{4 P_{02}}+\mathrm{i} \boldsymbol{\nu} \cdot \boldsymbol{v}_{2}\right)}{B_{01} P_{02}-B_{02} P_{01}} \\
& \times \exp \left(\frac{\pi^{2} \nu^{2}}{4 M}\right) .
\end{aligned}
$$



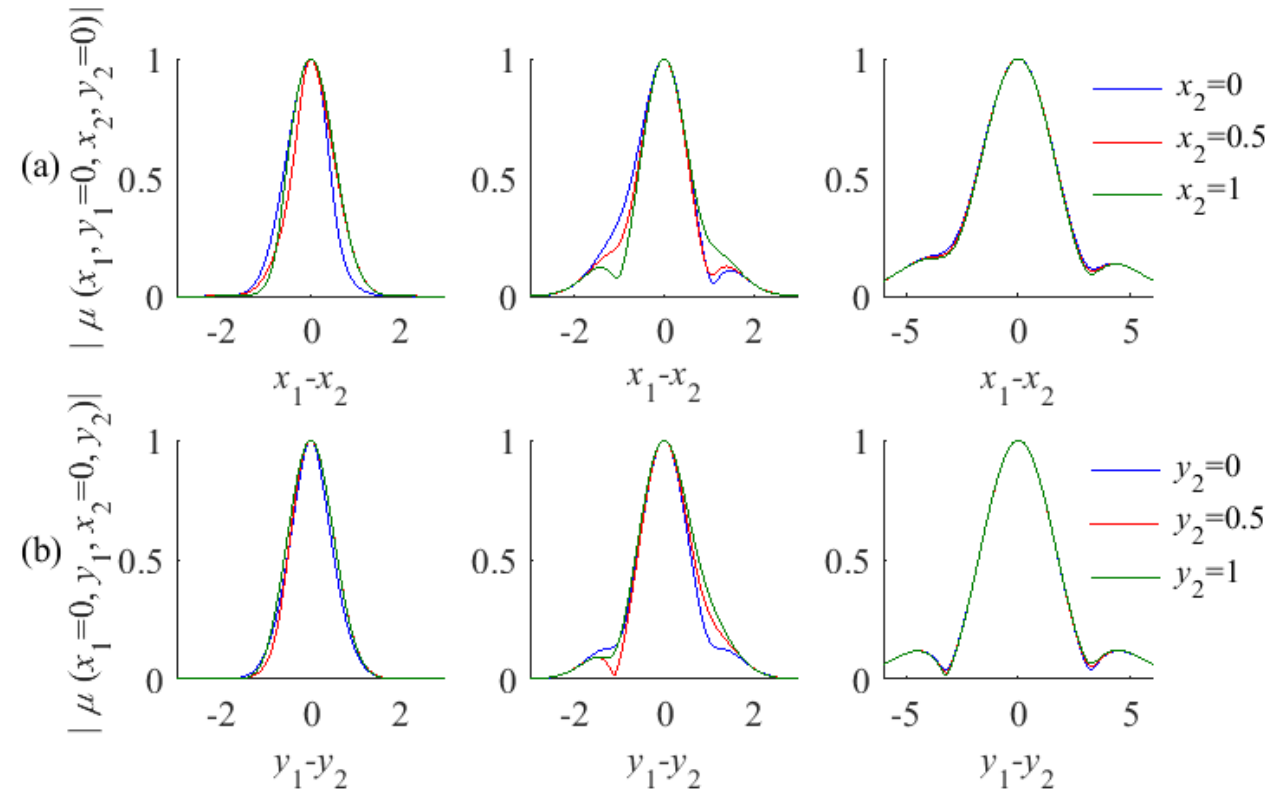

Figure 7: Absolute value of the degree of coherence (a) along $x_{1}$ direction for several $x_{2}$ values and (b) along $y_{1}$ direction for several $y_{2}$ values at different propagation distances: $z=0, z=z_{R 1}$, and $z=4 z_{R 1}$ from left to right. Other parameters are $\sigma_{01}=1, \sigma_{02}=0.85$, $\delta_{02}=0.5, u_{2}=0.1, B_{01}=1$, and $B_{02}=B_{02}^{(\max )}$.

Equation (43) shows that the CSD of the beam in the far field is of the Schell-model type, even though this does not happen at the source plane and for finite values of $z$. The intensity profile is Gaussianly shaped [Eq. (44)], while the degree of coherence, although not Gaussian [Eq. (45)], depends only on the difference of the coordinates of the two points.

Such feature of the degree of coherence is made evident in Fig. 7, where the modulus of $\mu$ is plotted for points along the $x$ and $y$ axes as a function of the variable $\left(x_{1}-x_{2}\right)$ and $\left(y_{1}-y_{2}\right)$, respectively, for several values of the propagation distance and for different values of $x_{2}$ or $y_{2}$. On increasing the propagation distance, less and less differences can be found among the shifted versions of the degree of coherence and, when $z$ is much greater than the Rayleigh distance, $\mu$ turns out to be practically shift invariant.

Figure 8 shows the absolute value of the far-field degree of coherence given in Eq. (45). It can be noted that it is nearly circularly symmetric, with higher values at the center followed by a ring of relatively low coherence sorrounded by another ring of higher degree of coherence. Except for a scale factor and differences along the low degree of coherence ring, this figure is quite similar to that in the lower right part of Fig. 5, as it could be expected.

Let us analyze the possibility of finding zero values of the far field degree of coherence for finite values of $\boldsymbol{\nu}$. The first exponential in the right-hand side of Eq. (45) is always real valued, while in the second term a complex factor appears. Then, the degree of coherence only can be zero if this complex factor becomes real, that is, if the argument 


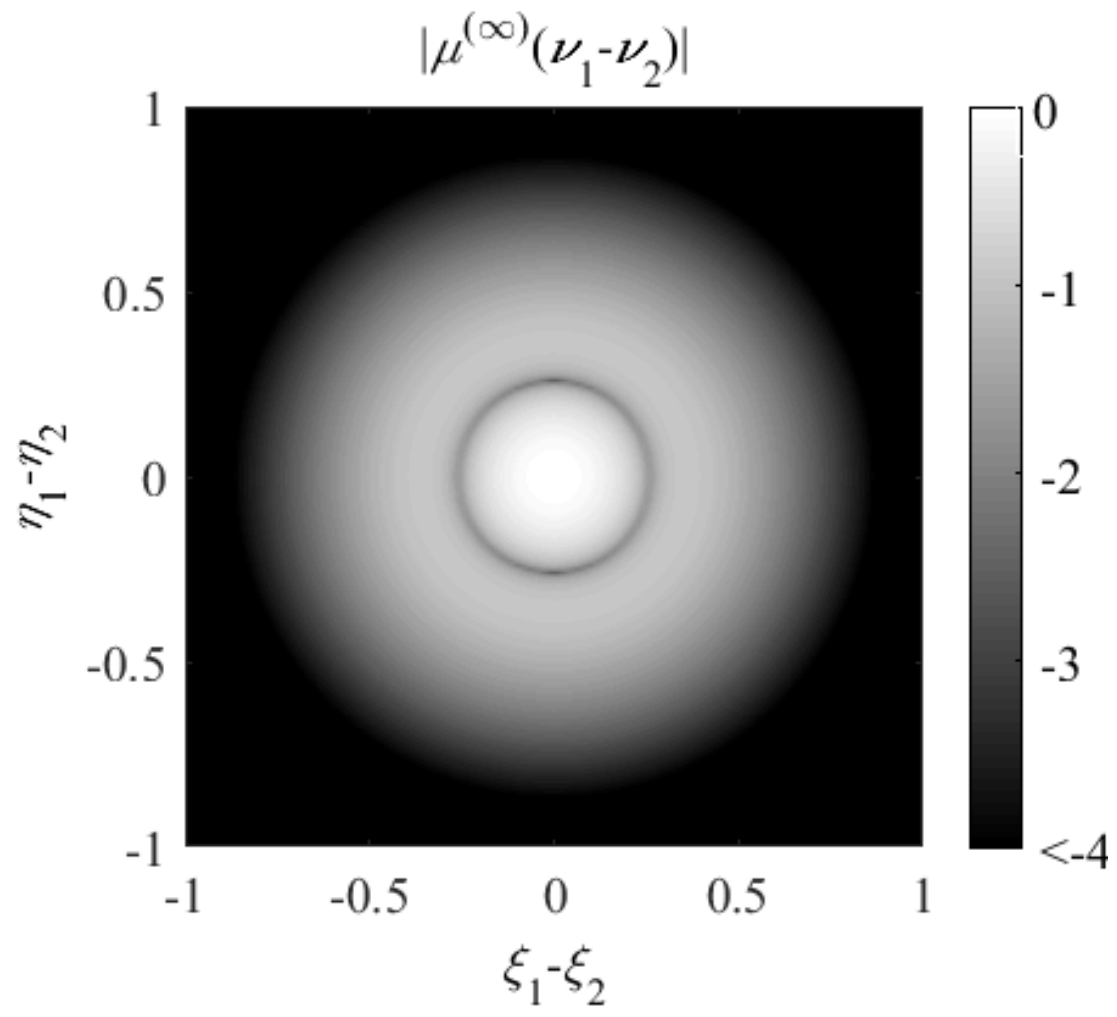

Figure 8: Absolute value of the degree of coherence in the far field, in logarithmic scale. $\eta$ and $\xi$ are the Cartesian coordinates of $\boldsymbol{\nu}$. Source parameters are $\sigma_{01}=1, \sigma_{02}=0.85$, $\delta_{02}=0.5, u_{2}=0.1, B_{01}=1$, and $B_{02}=B_{02}^{(\max )}$. 
of the complex exponential is of the form $\boldsymbol{\nu} \cdot \boldsymbol{v}_{2}=2 n \pi$, with integer $n$.

This surely happens if $\boldsymbol{v}_{2}=0$, i.e., if the two subtracted sources are not mutually displaced. In such a case, it is easily seen that $\mu^{(\infty)}(\boldsymbol{\nu})=0$ if

$$
B_{01} P_{02} \exp \left(-\frac{\pi^{2} \nu^{2}}{4 P_{01}}\right)=B_{02} P_{01} \exp \left(-\frac{\pi^{2} \nu^{2}}{4 P_{02}}\right)
$$

which gives rise to

$$
\nu^{2}=\frac{4}{\pi^{2}} \frac{P_{01} P_{02}}{P_{02}-P_{01}} \ln \left(\frac{B_{01}}{B_{02}} \frac{P_{02}}{P_{01}}\right) .
$$

This represents a circumference centered at the origin and with a radius defined by the source parameters. Note that conditions of Eq. (27) guarantee the positiveness of the right-hand side of Eq. (47).

Conversely, if $\boldsymbol{v}_{2} \neq 0$ the function $\mu^{(\infty)}(\boldsymbol{\nu})$ vanishes only in a discrete set of points of the $\boldsymbol{\nu}$-plane. In fact, in addition to Eq. (47), the condition $\boldsymbol{\nu} \cdot \boldsymbol{v}_{2}=2 n \pi$ (with integer $n$ ) has to be met. The latter condition identifies a set of straight lines orthogonal to the vector $\boldsymbol{v}_{2}$, evenly spaced with period $2 \pi / v_{2}$, whose intersections with the circumference correspond to the points where $\mu^{(\infty)}(\boldsymbol{\nu})=0$. Two of such points are always located along the line passing through the circumference center $(n=0)$ as can be observed in the bottom right part of Fig. 7. In Figure 8, it can be appreciated that the two intersection points between the $\eta$ axis and the ring of relatively low degree of coherence are darker than its surroundings. Additional intersection points are present if the radius of the circumference or the shift parameter $\boldsymbol{v}_{2}$ are large enough.

\section{Conclusions}

In this work a set of conditions under which the function obtained by subtracting two GSM CSD (arbitrarily displaced across the source plane) is itself a valid CSD has been found and discussed. The properties of the resulting CSD have then been studied both at the source plane and in propagation. It has been shown that the obtained CSD is not of the SM type, because the degree of coherence is not shift invariant, and the intensity profile is not Gaussian. This happens at any finite propagation distance. However, on approaching the far-field conditions, the degree of coherence tends to become shift invariant (although not Gaussian) and the intensity profile tends to become Gaussian. Finally, an experimental procedure to obtain this kind of CSDs has been proposed, making use of a $4 f$ telescopic optical system and amplitude filters.

\section{Acknowledgments}

This work has been partially supported by Spanish Ministerio de Economía y Competitividad under project FIS2013-46475. 


\section{References}

[1] Wolf E 2007 Introduction to the Theory of Coherence and Polarization of Light (Cambridge U. Press, Cambridge)

[2] Mandel L and Wolf E 1995 Optical Coherence and Quantum Optics (Cambridge U. Press, Cambridge)

[3] Gbur G and Visser T D 2010 Progress in Optics 55. 285-311 (Ed. E. Wolf, Elsevier, Amsterdam)

[4] Li Y, Guo J, Liu L, Wang T and Shao J 2013 Linear equations method for modal decomposition using intensity information J. Opt. Soc. Am. A 30 2502-2509

[5] Zhu S, Chen Y and Cai Y 2013 Experimental determination of the radius of curvature of an isotropic Gaussian Schell-model beam J. Opt. Soc. Am. A 30 171-176

[6] Singh M, Tervo J and Turunen J 2013 Elementary-field analysis of partially coherent beam shaping J. Opt. Soc. Am. A 30 2611-2617

[7] Huang Y and Zhang B 2013 Turbulence distance for laser beams propagating through non-Kolmogorov turbulence J. Opt. Soc. Am. A 30 2339-2346

[8] Alieva T, Rodrigo J A, Cámara A and Abramochkin E 2013 Partially coherent stable and spiral beams J. Opt. Soc. Am. A 30 2237-2243

[9] Yamazoe K, Mochi I and Goldberg K A 2014 Gradient descent algorithm applied to wavefront retrieval from through-focus images by an extreme ultraviolet microscope with partially coherent source J. Opt. Soc. Am. A 31 B34-B43

[10] Cai Y, Chen Y and Wang F 2014 Generation and propagation of partially coherent beams with nonconventional correlation functions: a review [Invited] J. Opt. Soc. Am. A 31 2083-2096

[11] Gbur G 2014 Partially coherent beam propagation in atmospheric turbulence [Invited] J. Opt. Soc. Am. A 31 2038-2045

[12] Martínez-Herrero R, Mejías P M and Piquero G 2009 Characterization of partially polarized light fields (Springer Series in Optical Sciences, 147, Springer-Verlag, Berlin)

[13] de Sande J C G, Santarsiero M, Piquero G and Gori F 2012 Longitudinal polarization periodicity of unpolarized light passing through a double wedge depolarizer Opt. Express $2027348-27360$

[14] Lahiri M and Wolf E 2013 Change in spatial coherence of light on refraction and on reflection J. Opt. Soc. Am. A 30 1107-1112

[15] Lahiri M and Wolf E 2013 Propagation of electromagnetic beams of any state of spatial coherence and polarization through multilayered stratified media J. Opt. Soc. Am. A 30 2547-2555

[16] Yuan Y, Du S, Dong Y, Wang F, Zhao C and Cai Y 2013 Nonparaxial propagation properties of a vector partially coherent dark hollow beam J. Opt. Soc. Am. A 30 $1358-1372$ 
[17] Raghunathan S B, Schouten H F and Visser T D 2013 Topological reactions of correlation functions in partially coherent electromagnetic beams J. Opt. Soc. Am. A 30 582-588

[18] Shu J, Chen Z and Pu J 2013 Radiation forces on a Rayleigh particle by highly focused partially coherent and radially polarized vortex beams J. Opt. Soc. Am. A 30 916-922

[19] Santarsiero M, de Sande J C G, Piquero G and Gori Franco 2013 Coherencepolarization properties of fields radiated from transversely periodic electromagnetic sources J. Opt. 15055701

[20] Craeye C, Withington S and Thomas C N 2014 Characteristic functions describing the power absorption response of periodic structures to partially coherent fields $J$. Opt. Soc. Am. A 31 1360-1368

[21] de Sande J C G, Piquero G, Santarsiero M and Gori F 2014 Partially coherent electromagnetic beams propagating through double-wedge depolarizers J. Opt. 16 035708

[22] Fernández-Pousa C R 2013 Nonstationary elementary-field light randomly triggered by Poisson impulses J. Opt. Soc. Am. A 30 932-940

[23] Voipio T, Setl T and Friberg A T 2013 Partial polarization theory of pulsed optical beams J. Opt. Soc. Am. A 30 71-81

[24] Voipio T, Setl T and Friberg A T 2013 Coherent-mode representation of partially polarized pulsed electromagnetic beams J. Opt. Soc. Am. A, 30, 2433-2443

[25] Mokhtarpour L and Ponomarenko S A 2013 Ultrashort pulse coherence properties in coherent linear amplifiers J. Opt. Soc. Am. A 30 627-630

[26] Dutta R, Korhonen M, Friberg A T, Genty G and Turunen J 2014 Broadband spatiotemporal Gaussian Schell-model pulse trains J. Opt. Soc. Am. A 31 637-643

[27] Riesz F and Nagy B S 1956 Functional Analysis (Blackie and Sons, London)

[28] Gori F and Santarsiero M 2007 Devising genuine spatial correlation functions Opt. Lett. 32 3531-3533

[29] Martínez-Herrero R, Mejías P M and Gori F 2009 Genuine cross-spectral densities and pseudo-modal expansions Opt. Lett. 34 1399-1401

[30] Sahin and Korotkova O 2012 Light sources generating far fields with tunable flat profiles Opt. Lett. 37 2970-2972

[31] Korotkova O, Sahin S and Shchepakina E 2012 Multi-Gaussian Schell-model beams J. Opt. Soc. Am. A 29 2159-2164

[32] Cang J, Fang X and Liu X 2013 Propagation properties of multi-Gaussian Schellmodel beams through ABCD optical systems and in atmospheric turbulence Optics \&5 Laser Technology 50, 65-70

[33] Santarsiero M, Piquero G, de Sande J C G and Gori F 2014 Difference of crossspectral densities Opt. Lett. 39, 1713-1716 (2014). 
[34] Gori F and Santarsiero M 2014 Difference of two Gaussian Schell-model cross-spectral densities Opt. Lett. 39 2731-2734

[35] Borghi R, Gori F, Guattari G and Santarsiero M 2015 Shape-invariant difference between two Gaussian Schell-model beams J. Opt. Soc. Am. A 32 790-796

[36] Gori F 1983 Mode propagation of the field generated by Collett-Wolf Schell-model sources Opt. Commun. 46 149-154

[37] Goodman J W 2005 Introduction to Fourier optics, 3rd Ed. (Roberts \& Company, Englewood)

[38] Gori F 1980 Collett- Wolf sources and multimode lasers Opt. Commun. 34 301-305

[39] Gbur G and Wolf E 2001 The Rayleigh range of Gaussian Schell-model beams $J$. Modern Opt. 48 1735-1741 